\title{
A nomogram for the predicting of survival in patients with esophageal squamous cell carcinoma undergoing definitive chemoradiotherapy
}

\author{
Peiliang Wang ${ }^{1,2}$, Maoqi Yang ${ }^{3}$, Xin Wang ${ }^{2,4}$, Zongxing Zhao ${ }^{1,2}$, Minghuan $\mathrm{Li}^{2}$, Jinming Yu ${ }^{1,2}$ \\ ${ }^{1}$ Cheeloo College of Medicine, Shandong University, Jinan, China; ${ }^{2}$ Department of Radiation Oncology, Shandong Cancer Hospital and Institute, \\ Shandong First Medical University and Shandong Academy of Medical Sciences, Jinan, China; ${ }^{3}$ School of Pharmacy, Yantai University, Yantai, \\ China; ${ }^{4}$ Shandong First Medical University \& Shandong Academy of Medical Sciences, Jinan, China \\ Contributions: (I) Conception and design: M Li, J Yu, P Wang; (II) Administrative support: None; (III) Provision of study materials or patients: M Li, \\ P Wang; (IV) Collection and assembly of data: P Wang, M Yang, X Wang, Z Zhao; (V) Data analysis and interpretation: P Wang; (VI) Manuscript \\ writing: All authors; (VII) Final approval of manuscript: All authors. \\ Correspondence to: Minghuan Li. Department of Radiation Oncology, Shandong Cancer Hospital and Institute, Shandong First Medical University \\ and Shandong Academy of Medical Sciences, Jiyan Road 440, Jinan 250117, China. Email: Sdlmh2014@163.com; Jinming Yu. Department of \\ Radiation Oncology, School of Medicine, Shandong University, Wenhua West Road 44, Jinan 250012, China; Department of Radiation Oncology, \\ Shandong Cancer Hospital and Institute, Shandong First Medical University and Shandong Academy of Medical Sciences, Jiyan Road 440, Jinan \\ 250117, China. Email: sdyujinming@126.com.
}

Background: Definitive chemoradiotherapy (dCRT) is widely accepted for esophageal squamous cell carcinoma (ESCC), although the outcomes can vary. Therefore, we aimed to develop a nomogram for the pre-treatment prediction of survival after dCRT for ESCC.

Methods: This retrospective study evaluated 204 patients (169 patients in a primary cohort and 35 patients in a validation cohort) who received dCRT for ESCC between July 2013 and June 2017.

Results: Pre-treatment parameters that predicted long-term survival in this setting were body mass index (BMI), absolute lymphocyte count (ALC), neutrophil-to-lymphocyte ratio (NLR), wall thickness, concurrent chemoradiotherapy, radiotherapy modality, and American Joint Committee on Cancer (AJCC) stage. The nomogram incorporated these factors and provided C-index values of 0.691 [95\% confidence interval (CI): $0.641-0.740]$ in the primary cohort and 0.816 (95\% CI: $0.700-0.932)$ in the validation cohort. The calibration curve analysis revealed that the nomogram had good ability to predict 2-year progression-free survival (PFS). The nomogram also performed better than the AJCC staging system by the C-index values (0.691 vs. 0.560$)$ and the area under the curve values (0.702 vs. 0.576). Decision curve analysis (DCA) also indicated that the nomogram had better clinical utility.

Conclusions: These results suggest that pre-treatment parameters may help predict the efficacy of dCRT for ESCC. Furthermore, as the nomogram provided better prognostic accuracy than the AJCC staging system, the nomogram may be useful in clinical practice for prognostication among patients who are going to receive dCRT for ESCC.

Keywords: Chemoradiotherapy; definitive; esophageal squamous cell carcinoma (ESCC); long-term survival; nomogram; prognostication

Submitted Feb 09, 2020. Accepted for publication Oct 23, 2020.

doi: $10.21037 /$ atm-20-1460

View this article at: http://dx.doi.org/10.21037/atm-20-1460 


\section{Introduction}

Esophageal cancer is the sixth most deadly malignancy (1), and the most common type in Asia is esophageal squamous cell carcinoma (ESCC), whereas adenocarcinoma is currently more common in Australia, the UK, the USA, and some western European countries (2). Previous studies have indicated that chemoradiotherapy can provide a median survival time of 11-22 months and a 5-year survival rate of $27 \%$, which are similar to the outcomes after surgery (3-7). Thus, definitive chemoradiotherapy (dCRT) has become a widely accepted treatment for esophageal cancer, due to the less adverse effects (8). However, outcomes can vary broadly among patients with the same stage of ESCC, as survival is influenced by multiple factors. Furthermore, to the best of our knowledge, few studies have investigated whether pre-treatment clinical parameters can be used to predict survival among patients with ESCC who are undergoing dCRT. Therefore, the present study aimed to identify pretreatment factors that could be used to predict survival after dCRT for ESCC. Moreover, this study aimed to develop a prognostic nomogram using these factors, and to compare that nomogram's accuracy to that of the American Joint Committee on Cancer (AJCC) staging system. We present the following article in accordance with the TRIPOD reporting checklist (available at http://dx.doi.org/10.21037/ atm-20-1460).

\section{Methods}

\section{Patients}

The study was conducted in accordance with the Declaration of Helsinki (as revised in 2013) and approved by our institutional review board (SDTHEC201901006). The requirement for informed consent was waived based on the study's retrospective analysis of secured patient data. The results of this study did not affect the patients' future management.

This study included 204 patients from our registry who received dCRT for ESCC between July 2013 and June 2017. To minimize heterogeneity, patients were required to be $<80$ years old and to have stage II-IVa squamous cell carcinoma of the thoracic esophagus, based on the eighth edition of the AJCC staging system [2017]. The other eligibility criteria were good general condition (Eastern Cooperative Oncology Group performance status of 0 or 1), normal liver function, normal renal function and normal bone marrow. The exclusion criteria were gastric cardia infiltration, distant or supraclavicular lymph node metastasis, tracheobronchial involvement, respiratory insufficiency, Child-Pugh class B or $\mathrm{C}$ cirrhosis, and symptomatic coronary heart disease. To develop the nomogram, we divided the patients into a primary cohort (169 patients who were treated between July 2013 and December 2016) and a validation cohort (35 patients who were treated between January 2017 and June 2017).

The patients' characteristics were collected from their medical records and included age, sex, and carcinoembryonic antigen concentration. Two radiologists with $>10$ years of experience separately reviewed the medical imaging records and collected data regarding tumor length, tumor location, $\mathrm{X}$-ray type, minimum stenosis diameter based on barium swallow or endoscopic ultrasound findings, wall thickness, esophageal diameter, tumor status, and lymph node status. Missing values for the 27 clinical characteristics were replaced by the average value for the corresponding variable.

\section{Treatment and follow-up}

The patients underwent treatment using three-dimensional conformal radiotherapy or intensity-modulated radiotherapy (dose: 50-68 Gy), and were categorized according to whether their chemotherapy was provided concurrently (Table 1). The progression-free survival (PFS) interval was calculated from the end of radiotherapy to the first instance of recurrence, metastasis, or death.

\section{Factor selection and construction of nomogram}

Univariate Cox regression analyses were performed to identify potential prognostic factors. Variable selection was refined using the least absolute shrinkage and selection operator (LASSO) method, which is suitable for regression analysis of high-dimensional data with multicollinearity (9-12). A nomogram was constructed based on the results of the LASSO Cox regression model (13). The nomogram was subjected to bootstrapping validation (500 bootstrap resamples) to calculate a relatively corrected C-index. A calibration curve was applied to assess the nomogram and the area under the curve (AUC) was used to quantify the discriminative performance. The Hosmer-Lemeshow test was used to evaluate the nomogram's goodness-of-fit.

\section{Internal validation}

Internal validation was performed using the calibration 
Table 1 Characteristics of patients in the primary and validation cohorts

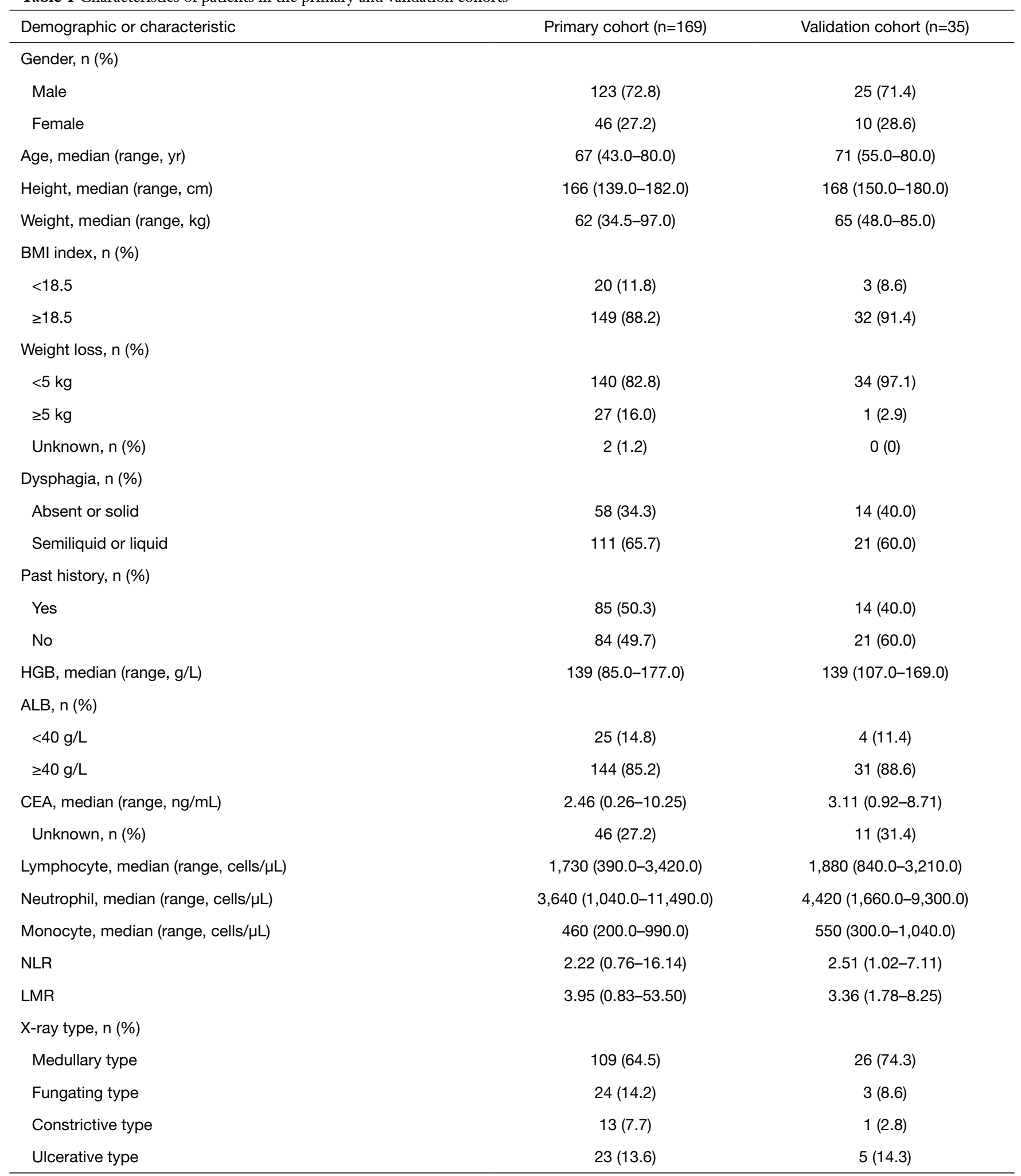

Table 1 (continued) 
Table 1 (continued)

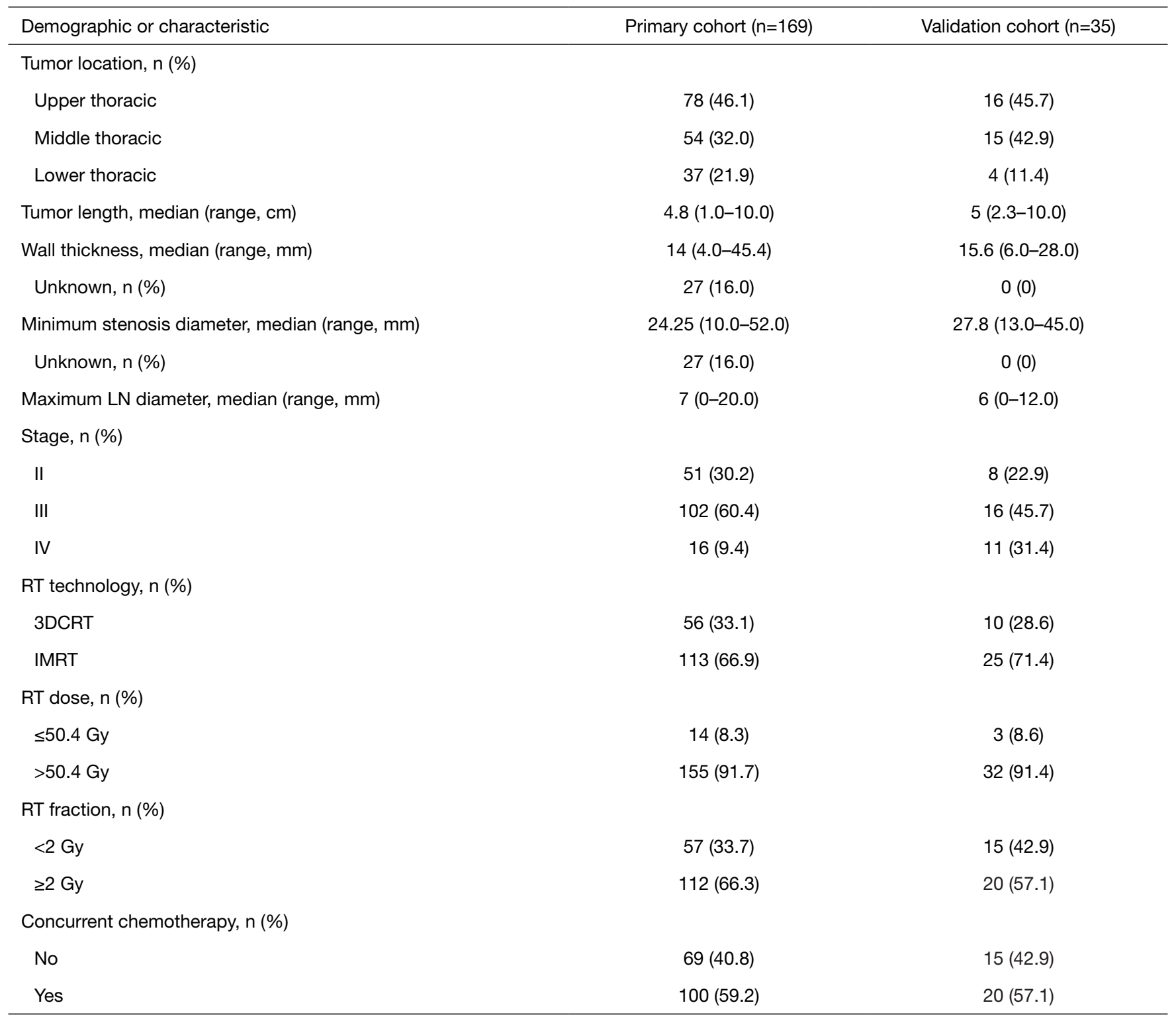

BMI, body mass index; HGB, hemoglobin; ALB, albumin; CEA, carcinoembryonic antigen; NLR, neutrophil-to-lymphocyte ratio; LMR, lymphocyte-to-monocyte ratio; RT, radiotherapy; 3D-CRT, three-dimensional conformal radiotherapy; IMRT, intensity modulated radiotherapy.

curve and AUC analysis in the validation cohort.

\section{Clinical utility}

Decision curve analysis (DCA) was performed for all subjects to examine the nomogram's clinical utility based on net benefits that were calculated at a series of threshold probabilities (14).

\section{Statistical analysis}

The Cox regression model with LASSO penalties was subjected to a 10 -fold cross-validation method based on minimum criteria. Detailed descriptions of the LASSO algorithm and DCA are provided in the figure legends. The "glmnet" and "survival" packages in R statistic software were used for the LASSO Cox regression analysis, and the "car" package was used to calculate the variance inflation 


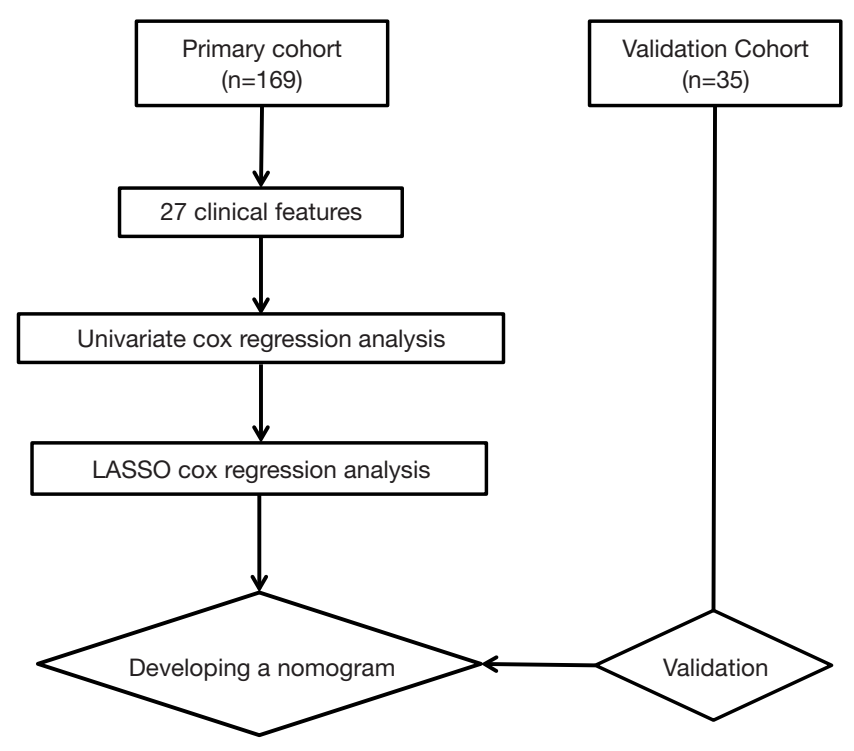

Figure 1 The flowchart of study procedure.

factors. The "pROC" package was applied to plot the receiver operating characteristic curve and the "rms" package was used for the nomogram's construction and calibration plotting. The DCA was performed using the "dca.R" package. Differences were considered significant in the univariate Cox regression analyses at two-sided $\mathrm{P}$-values of $<0.1$. All statistical analyses were performed using R software (version 3.4.4, http://www.R-project. org), EmpowerStats software (www.empowerstats.com, $\mathrm{X} \& Y$ Solutions Inc., Boston, MA) and IBM SPSS software (version 21.0; IBM Corp., Armonk, NY, USA).

\section{Results}

\section{Clinical characteristics}

The study flowchart is presented in Figure 1. The 204 eligible patients were assigned to a primary cohort (169 patients who received treatment between July 2013 and December 2016) and a validation cohort (35 patients who received treatment between January 2017 and June 2017). The patients' characteristics are shown Table 1 . The primary and validation cohorts had similar clinical characteristics and 2-year PFS rates $(\mathrm{P}=0.381)$.

\section{Survival and treatment failure patterns}

The median follow-up time was 42.0 months [95\% confidence interval (CI): 38.4-45.6 months] and the estimated median PFS time was 22.0 months (95\% CI: 12.7-31.3 months). The estimated PFS rates were 58.8\% at 1 year and $45.6 \%$ at 2 years. The treatment failure manifested as local failure (73\% of cases) or distant failure (22\% of cases). The locoregional recurrences involved local failure at the primary tumor site ( $86 \%$ of cases) and the lymph nodes ( $21 \%$ of cases).

\section{Factor selection and model construction}

The univariate Cox regression analyses revealed that 12 of the 27 pre-treatment clinical parameters were significantly associated with PFS in the primary cohort (Table 2). According to the results of LASSO cox regression model, the nomogram ultimately included body mass index (BMI), absolute lymphocyte count (ALC), neutrophilto-lymphocyte ratio (NLR), wall thickness, concurrent chemoradiotherapy, radiotherapy modality, and AJCC stage (Figure 2A,B,C, Table 2). No collinearity was observed by analyzing the variance inflation factors for those 7 parameters (Table S1).

\section{Performance evaluation}

The nomogram's performance was evaluated in the primary cohort and the C-index for PFS was 0.691 (95\% CI: $0.641-0.740$ ), which was confirmed by 1,000 bootstrap 
A

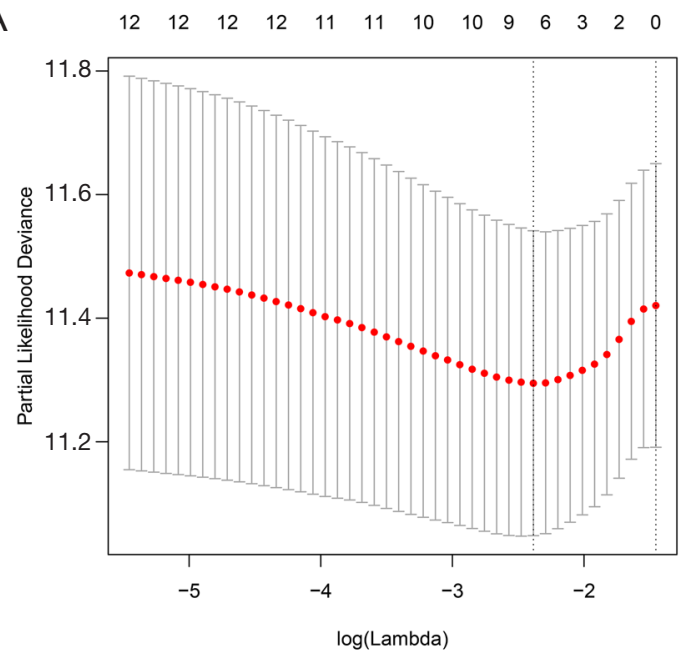

B

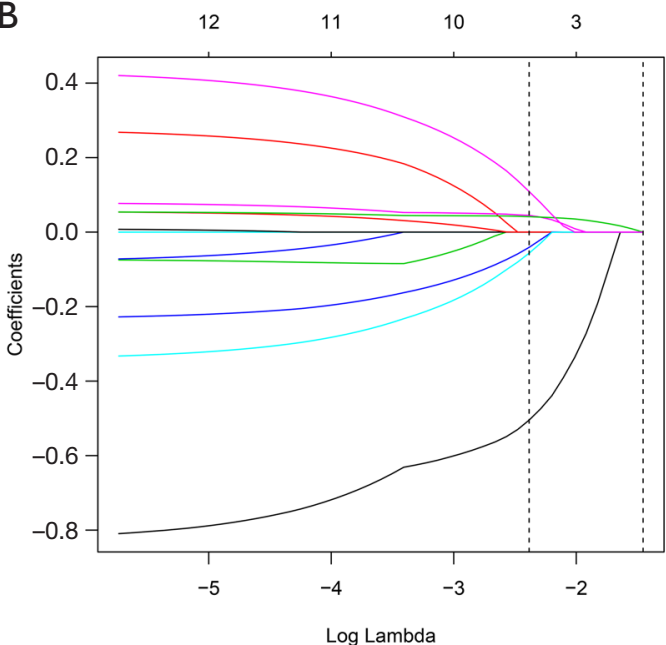

C

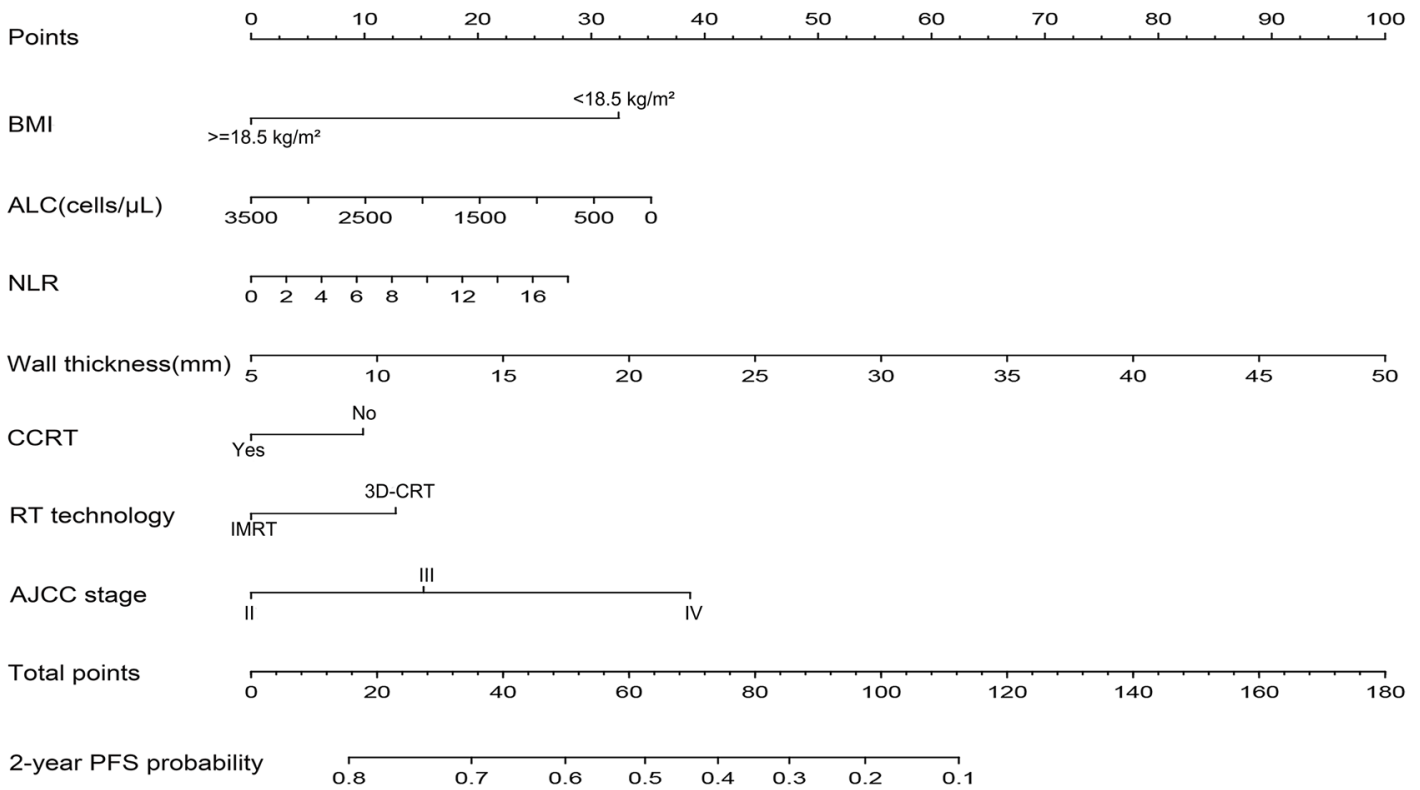

Figure 2 Feature selection using the least absolute shrinkage and selection operator (LASSO) Cox regression model (A,B). (A) Tuning parameter $(\lambda)$ selection in the LASSO model via 10 -fold cross-validation based on minimum criteria. The partial likelihood deviance curve was plotted versus $\log (\lambda)$. Dotted vertical lines define the optimal values of $\lambda$, where the model provides its best fit to the data. The optimal $\lambda$ value of 0.092 with $\log (\lambda)=-2.38$ was selected. (B) LASSO coefficient profiles of the 12 features. A coefficient profile plot was produced against the $\log (\lambda)$ sequence. Vertical line was drawn at the value selected using 10 -fold cross-validation, where optimal lambda resulted in seven features with nonzero coefficients. (C) Nomogram. BMI, body mass index; ALC, absolute lymphocyte count; NLR, neutrophil-tolymphocyte ratio; CCRT, concurrent chemoradiotherapy; AJCC stage, American Joint Committee on Cancer staging system. 
Table 2 Univariate cox regression model and LASSO Cox regression model for PFS

\begin{tabular}{|c|c|c|c|c|}
\hline Characteristics & \multicolumn{3}{|c|}{ Univariate analysis (PFS) } & $\frac{\text { LASSO Cox }}{\text { Coefficient }}$ \\
\hline BMI (<18.5 vs. $\left.\geq 18.5 \mathrm{~kg} / \mathrm{m}^{2}\right)$ & 0.353 & $0.211-0.591$ & $<0.001$ & -0.505 \\
\hline Weight loss ( $\geq 5 \%$ vs. $<5 \%)$ & 1.668 & $1.020-2.725$ & 0.041 & - \\
\hline CEA & 1.103 & $0.982-1.240$ & 0.099 & - \\
\hline ALC & 1.000 & $0.999-1.000$ & 0.030 & $<0.001$ \\
\hline NLR & 1.189 & $1.079-1.311$ & $<0.001$ & 0.045 \\
\hline Tumor location & & & & - \\
\hline \multicolumn{5}{|l|}{ Upper thoracic } \\
\hline Tumor length & 1.129 & $1.025-1.244$ & 0.014 & - \\
\hline Wall thickness & 1.079 & $1.044-1.114$ & $<0.001$ & 0.041 \\
\hline Concurrent chemotherapy (no vs. yes) & 0.683 & $0.461-1.013$ & 0.058 & -0.057 \\
\hline RT technology (3D-CRT vs. IMRT) & 0.619 & $0.415-0.925$ & 0.019 & -0.041 \\
\hline AJCC stage & & & & 0.109 \\
\hline \multicolumn{5}{|l|}{ II } \\
\hline III & 1.400 & $0.879-2.231$ & 0.157 & \\
\hline IVa & 2.478 & $1.283-4.786$ & 0.007 & \\
\hline
\end{tabular}

LASSO, least absolute shrinkage and selection operator; PFS, progression-free survival; BMI, body mass index; CEA, carcinoembryonic antigen; ALB, albumin; ALC, absolute lymphocyte counts; NLR, neutrophil-to-lymphocyte ratio; RT, radiotherapy; 3D-CRT, three-dimensional conformal radiotherapy; IMRT, intensity modulated radiotherapy.

resamples. The calibration curve for 2-year PFS suggested that the nomogram's predictive ability was aligned with the actual clinical outcomes (Figure $3 A$ ). The AUC of 0.702 for 2-year PFS also indicated that the nomogram had good discriminative ability (Figure 3B). The Hosmer-Lemeshow test revealed good calibration, with a non-significant result $(\mathrm{P}=0.201)$.

\section{Validating predictive accuracy}

The calibration curve also revealed good agreement between the predicted 2-year PFS probability and the actual clinical outcomes (Figure 3C). The predictive accuracy was also evaluated in the validation cohort, which revealed a C-index value of 0.816 (95\% CI: 0.700-0.932) and an AUC of 0.869 for predicting 2-year PFS (Figure 3D, Table S2).
Comparing the predictive accuracies of the nomogram and AfCC staging system

The eighth edition of the AJCC staging system generally provides good prognostic stratification, although the stratification was not satisfactory for patients with stage II-III ESCC (Figure $4 A, B$ ). In the primary cohort, our nomogram provided better ability to predict PFS than the AJCC staging system based on the C-index values (0.691 vs. 0.560, $\mathrm{P}=0.016$ ) (Table S2). Furthermore, the nomogram had better discriminative ability than the AJCC staging system according to the higher AUC value for 2-year PFS (0.702 vs. 0.576) (Figure 4C, Table S2). When considered together, these results suggested that the nomogram might be more effective than the AJCC staging system for predicting PFS after dCRT for ESCC.

The 2-year DCA curves also revealed that the nomogram 
A

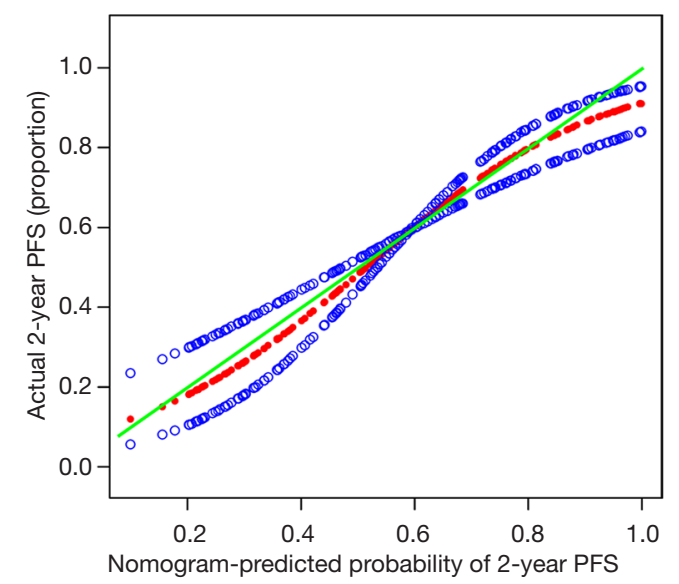

C

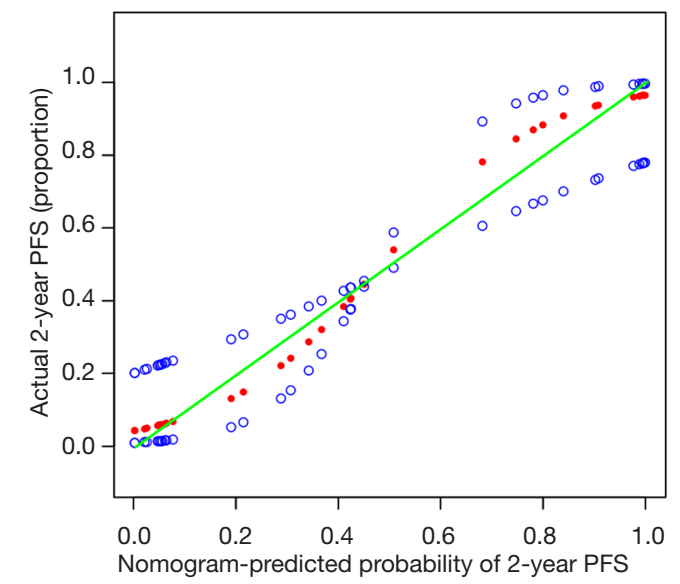

B

Method: NNE, time $=24, \mathrm{AUC}=0.702$

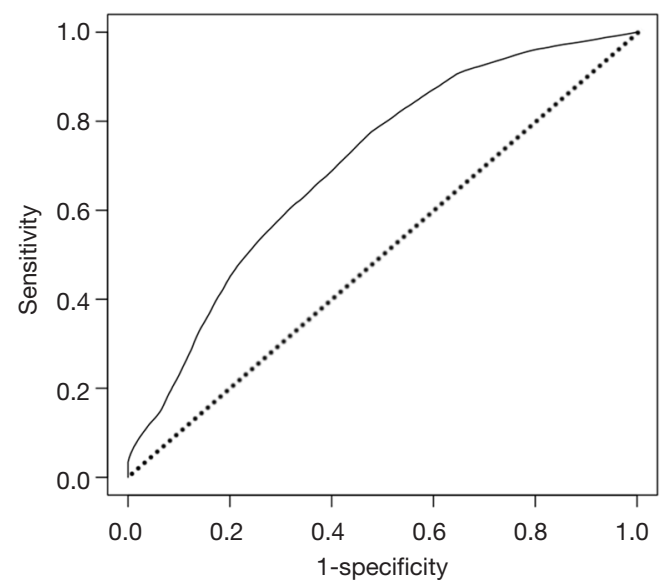

Dethod: NNE, time $=24$, AUC $=0.869$

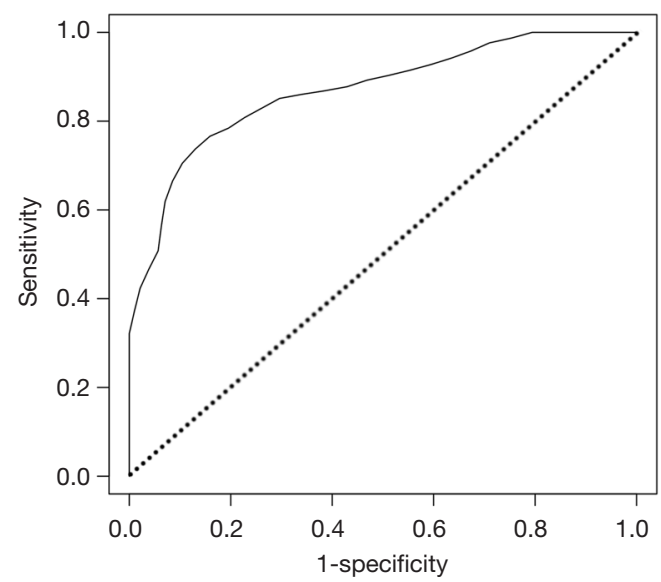

Figure 3 Performance of the nomogram (A,C). Calibration plots showing the consistency between the predicted probabilities based on the nomogram and actual values in the primary cohort (A) and validation cohort (C). The diagonal green solid line represents a perfect prediction by an ideal model. The red dotted line represents the performance of the nomogram, of which a closer fit to the diagonal green solid line represents a better prediction. The blue dotted lines represent $95 \%$ CIs of the nomogram. ROC curves presenting the predictive power of the nomogram for 2-year PFS in primary cohort (B) and validation cohort (D). ROC, receiver operator characteristic; PFS, progression-free survival; NNE, Nearest neighbor estimate; AUC, area under the curve; time =24 months (2 years).

had better clinical performance than the AJCC staging system among all study subjects (Figure 5).

\section{Discussion}

The present study evaluated 27 pre-treatment clinical parameters for patients who received dCRT for ESCC, including their general condition, hematological indicators, imaging findings, and treatment parameters. The LASSO Cox model was used to identify prognostic factors because it is suitable for analysis of data with multicollinearity, it is more robust than stepwise regression analysis, and can avoid overfitting (15). Based on the results of that analysis, the nomogram ultimately included BMI, ALC, NLR, wall thickness, concurrent chemoradiotherapy, radiotherapy modality, and AJCC stage.

Previous studies have indicated that nutritional support can help limit the adverse effects of radiotherapy and improve outcomes among patients with colorectal, head, and neck cancers $(16,17)$. However, the baseline nutritional status is not generally considered for patients with esophageal cancer who are receiving dCRT. The present 


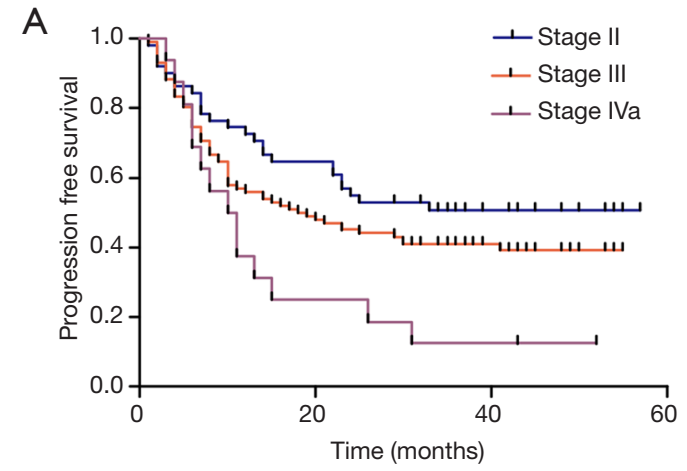

C Nomogram (black) vs. AJCC stage (Red)

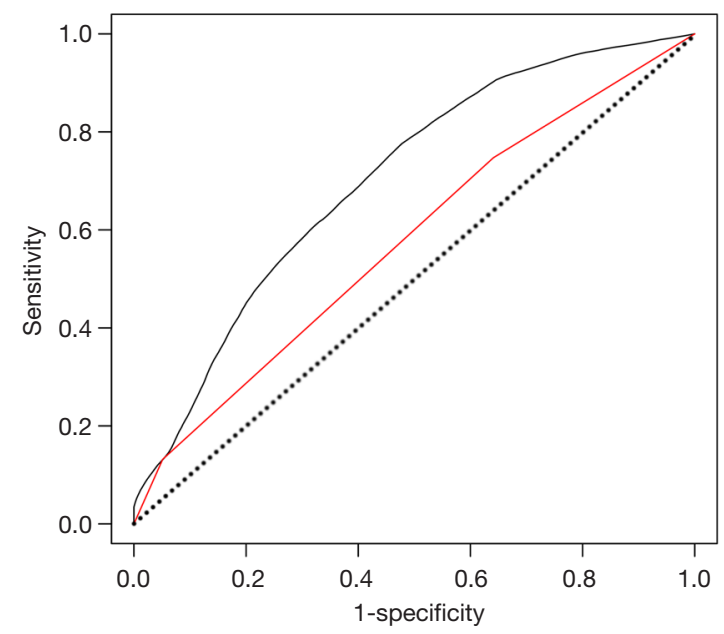

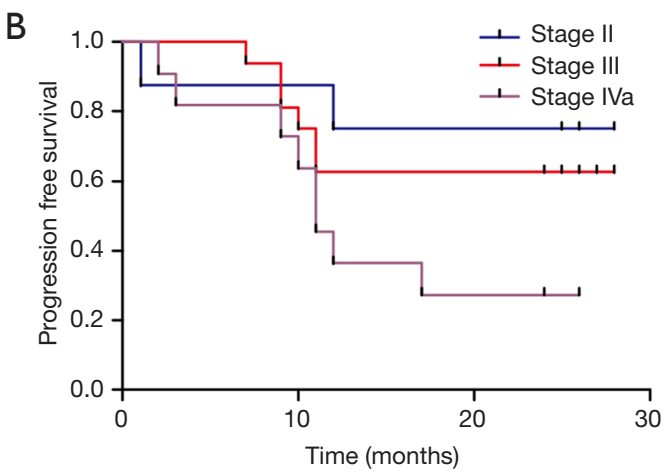




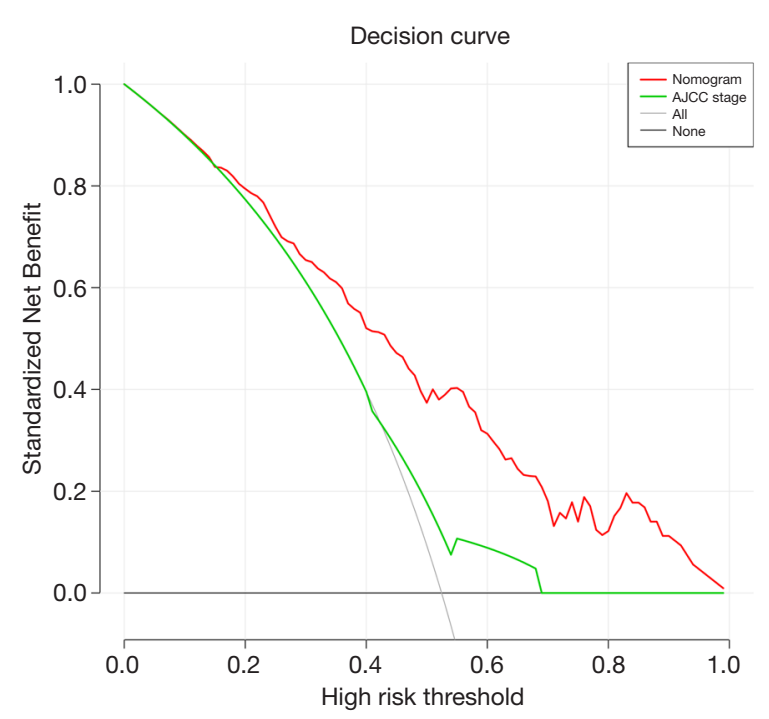

Figure 5 Decision curve analysis for the nomogram and the AJCC stage. The $\mathrm{x}$-axis represents the high risk threshold (threshold probability). The y-axis represents the standardized net benefit. The gray line represents the hypothesis that all patients had 2-year PFS. The black line represents the hypothesis that no patients had 2-year PFS. If one model achieves the highest net benefit compared with other models or any simple strategies at any given threshold, it is of clinical significance. PFS, progression-free survival.

associated with the response to chemoradiotherapy among patients with T3-4 ESCC (25). Similarly, the present study revealed that the response to chemoradiotherapy and treatment outcomes were significantly correlated with the pre-treatment maximal esophageal wall thickness.

Several studies have demonstrated that, relative to sequential CRT, concurrent CRT can provide a significantly higher survival rate among patients with esophageal cancer. Although it had been suggested that concurrent CRT would be associated with more adverse effects, clinical experience has revealed that concurrent CRT is well-tolerant and can be recommended to patients with esophageal cancer. Retrospective studies have also suggested that the dosimetric advantage of intensity-modulated radiotherapy, relative to three-dimensional conformal radiotherapy, was related to improved clinical outcomes (26).

The AJCC staging system provides impressive prognostic value for some patients with ESCC, although it was not specifically developed for the prediction of post-treatment outcomes (27). Moreover, among patients undergoing dCRT for ESCC, the predictive accuracy of the AJCC staging system might be affected by other important factors, such as nutritional status, hematological biomarkers, and therapeutic regimen. Thus, we developed a comprehensive nomogram that evaluates various important pre-treatment parameters, such as nutritional status (BMI), radiographic characteristics (wall thickness), immune and inflammatory biomarkers (ALC and NLR), therapeutic regimen (concurrent chemotherapy and radiotherapy modality), and AJCC stage. The performance of the nomogram was satisfactory based on the calibration curve and C-index values (primary cohort: 0.691 , validation cohort: 0.816 ). Moreover, we found that the nomogram was better than the AJCC staging system for predicting survival outcomes, and the DCA results also demonstrated that our nomogram provided greater clinical value than the AJCC grading system. In addition, Factors included in the nomogram can be judged during a pre-treatment clinical examination, which would be useful in clinical practice for predicting survival after dCRT for ESCC. Finally, most patients who do not experience recurrence within 2 years after dCRT are likely to remain without recurrence during the first 5 years after treatment (19). Thus, our model may help predict the likelihood of long-term survival (28).

The present study has several limitations. First, we did not consider all potentially relevant characteristics, such as genetic markers, tumor differentiation status, and pulmonary function. Second, the specific chemotherapy regimens were not analyzed. Third, the retrospective study design is associated with possible sources of bias. Thus, multicenter studies with larger sample sizes are needed to validate our nomogram, identify areas for improvement, and generate additional evidence regarding its clinical application.

\section{Conclusions}

We developed a nomogram for ESCC that included BMI, ALC, NLR, wall thickness, concurrent chemoradiotherapy, radiotherapy modality, and AJCC stage. This nomogram was useful for predicting 2-year PFS among patients who were receiving dCRT for ESCC. Therefore, it may be prudent to consider these pre-treatment parameters when planning dCRT for ESCC patients. Our new nomogram may be useful for predicting clinical and survival outcomes among patients who are going to receive dCRT for ESCC.

\section{Acknowledgments}

Funding: This study was funded by the Natural Science 
Foundation of China (NSFC 81672995) and the Key Research and Development Program of Shandong Province (2016GSF201133).

\section{Footnote}

Reporting Checklist: The authors have completed the TRIPOD reporting checklist. Available at http://dx.doi. org/10.21037/atm-20-1460

Data Sharing Statement: Available at http://dx.doi. org/10.21037/atm-20-1460

Conflicts of Interest: All authors have completed the ICMJE uniform disclosure form (available at http://dx.doi. org/10.21037/atm-20-1460). The authors have no conflicts of interest to declare.

Ethical Statement: The authors are accountable for all aspects of the work in ensuring that questions related to the accuracy or integrity of any part of the work are appropriately investigated and resolved. The study was conducted in accordance with the Declaration of Helsinki (as revised in 2013) and approved by our institutional review board (SDTHEC201901006). The requirement for informed consent was waived based on the study's retrospective analysis of secured patient data.

Open Access Statement: This is an Open Access article distributed in accordance with the Creative Commons Attribution-NonCommercial-NoDerivs 4.0 International License (CC BY-NC-ND 4.0), which permits the noncommercial replication and distribution of the article with the strict proviso that no changes or edits are made and the original work is properly cited (including links to both the formal publication through the relevant DOI and the license). See: https://creativecommons.org/licenses/by-nc-nd/4.0/.

\section{References}

1. Bray F, Ferlay J, Soerjomataram I, et al. Global cancer statistics 2018: GLOBOCAN estimates of incidence and mortality worldwide for 36 cancers in 185 countries. CA Cancer J Clin 2018;68:394-424.

2. Hongo M, Nagasaki Y, Shoji T. Epidemiology of esophageal cancer: Orient to Occident. Effects of chronology, geography and ethnicity. J Gastroenterol Hepatol 2009;24:729-35.
3. Algan O, Coia LR, Keller SM, et al. Management of adenocarcinoma of the esophagus with chemoradiation alone or chemoradiation followed by esophagectomy: results of sequential nonrandomized phase II studies. Int J Radiat Oncol Biol Phys 1995;32:753-61.

4. al-Sarraf M, Martz K, Herskovic A, et al. Progress report of combined chemoradiotherapy versus radiotherapy alone in patients with esophageal cancer: an intergroup study. J Clin Oncol 1997;15:277-84.

5. Ilson D, Lordick F. Definitive or neoadjuvant chemoradiotherapy for squamous cell oesophageal cancer? Lancet Oncol 2018;19:1285-6.

6. Karran A, Blake P, Chan D, et al. Propensity score analysis of oesophageal cancer treatment with surgery or definitive chemoradiotherapy. Br J Surg 2014;101:502-10.

7. Wang BY, Wu SC, Chen HC, et al. Survival after neoadjuvant chemoradiotherapy and oesophagectomy versus definitive chemoradiotherapy for patients with oesophageal squamous cell carcinoma. Br J Surg 2019;106:255-62.

8. Cooper JS, Guo MD, Herskovic A, et al. Chemoradiotherapy of locally advanced esophageal cancer: long-term follow-up of a prospective randomized trial (RTOG 85-01). Radiation Therapy Oncology Group. JAMA 1999;281:1623-7.

9. Friedman J, Hastie T, Tibshirani R. Regularization Paths for Generalized Linear Models via Coordinate Descent. J Stat Softw 2010;33:1-22.

10. Sauerbrei W, Royston P, Binder H. Selection of important variables and determination of functional form for continuous predictors in multivariable model building. Stat Med 2007;26:5512-28.

11. Tibshirani R. The lasso method for variable selection in the Cox model. Stat Med 1997;16:385-95.

12. Wang L, Shen J, Thall PF. A Modified Adaptive Lasso for Identifying Interactions in the Cox Model with the Heredity Constraint. Stat Probab Lett 2014;93:126-33.

13. Touijer K, Scardino PT. Nomograms for staging, prognosis, and predicting treatment outcomes. Cancer 2009;115:3107-11.

14. Vickers AJ, Cronin AM, Elkin EB, et al. Extensions to decision curve analysis, a novel method for evaluating diagnostic tests, prediction models and molecular markers. BMC Med Inform Decis Mak 2008;8:53.

15. Hepp T, Schmid M, Gefeller O, et al. Approaches to Regularized Regression - A Comparison between Gradient Boosting and the Lasso. Methods Inf Med 2016;55:422-30. 
16. Ravasco P, Monteiro-Grillo I, Marques Vidal P, et al. Impact of nutrition on outcome: a prospective randomized controlled trial in patients with head and neck cancer undergoing radiotherapy. Head Neck 2005;27:659-68.

17. Ravasco P, Monteiro-Grillo I, Vidal PM, et al. Dietary counseling improves patient outcomes: a prospective, randomized, controlled trial in colorectal cancer patients undergoing radiotherapy. J Clin Oncol 2005;23:1431-8.

18. Khanna P, Blais N, Gaudreau PO, et al. Immunotherapy Comes of Age in Lung Cancer. Clin Lung Cancer 2017;18:13-22.

19. Vartolomei MD, Porav-Hodade D, Ferro M, et al. Prognostic role of pretreatment neutrophil-to-lymphocyte ratio (NLR) in patients with non-muscle-invasive bladder cancer (NMIBC): A systematic review and meta-analysis. Urol Oncol 2018;36:389-99.

20. Xu J, Ni C, Ma C, et al. Association of neutrophil/ lymphocyte ratio and platelet/lymphocyte ratio with ER and PR in breast cancer patients and their changes after neoadjuvant chemotherapy. Clin Transl Oncol 2017;19:989-96.

21. He JR, Shen GP, Ren ZF, et al. Pretreatment levels of peripheral neutrophils and lymphocytes as independent prognostic factors in patients with nasopharyngeal carcinoma. Head Neck 2012;34:1769-76.

22. Aliustaoglu M, Bilici A, Seker M, et al. The association of pre-treatment peripheral blood markers with survival in patients with pancreatic cancer. Hepatogastroenterology

Cite this article as: Wang $\mathrm{P}$, Yang $M$, Wang $\mathrm{X}$, Zhao $\mathrm{Z}$, Li M, Yu J. A nomogram for the predicting of survival in patients with esophageal squamous cell carcinoma undergoing definitive chemoradiotherapy. Ann Transl Med 2021;9(3):233. doi: $10.21037 /$ atm-20-1460
2010;57:640-5.

23. Davuluri R, Jiang W, Fang P, et al. Lymphocyte Nadir and Esophageal Cancer Survival Outcomes After Chemoradiation Therapy. Int J Radiat Oncol Biol Phys 2017;99:128-35.

24. Swisher SG, Maish M, Erasmus JJ, et al. Utility of PET, CT, and EUS to identify pathologic responders in esophageal cancer. Ann Thorac Surg 2004;78:1152-60; discussion -60 .

25. Li SH, Rau KM, Lu HI, et al. Pre-treatment maximal oesophageal wall thickness is independently associated with response to chemoradiotherapy in patients with T3-4 oesophageal squamous cell carcinoma. Eur J Cardiothorac Surg 2012;42:958-64.

26. Xi M, Lin SH. Recent advances in intensity modulated radiotherapy and proton therapy for esophageal cancer. Expert Rev Anticancer Ther 2017;17:635-46.

27. Rice TW, Rusch VW, Ishwaran H, et al. Cancer of the esophagus and esophagogastric junction: data-driven staging for the seventh edition of the American Joint Committee on Cancer/International Union Against Cancer Cancer Staging Manuals. Cancer 2010;116:3763-73.

28. Teoh AY, Chiu PW, Yeung WK, et al. Long-term survival outcomes after definitive chemoradiation versus surgery in patients with resectable squamous carcinoma of the esophagus: results from a randomized controlled trial. Ann Oncol 2013;24:165-71. 
Supplementary

Table S1 Multicollinearity analysis: VIF test

\begin{tabular}{lc}
\hline Characteristics & VIF(PFS) \\
\hline BMI & 1.0 \\
ALC & 1.4 \\
NLR & 1.3 \\
Wall thickness & 1.1 \\
Concurrent chemotherapy & 1.1 \\
RT technology & 1.1 \\
AJCC stage & 1.1 \\
\hline
\end{tabular}

BMI, body mass index; ALC, absolute lymphocyte counts; NLR, neutrophil-to-lymphocyte ratio; RT, radiotherapy.

Table S2 The value of C-index or AUC

\begin{tabular}{lcc}
\hline \multirow{2}{*}{ Groups } & \multicolumn{2}{c}{ Content } \\
\cline { 2 - 3 } & C-index & AUC \\
\hline Primary cohort & 0.691 & 0.702 \\
Validation cohort & 0.816 & 0.869 \\
AJCC staging system & 0.560 & 0.576 \\
\hline
\end{tabular}

C-index, Harrell's concordance indices; AUC, area under curve. 